\title{
Crossing the Threshold: the role of density dependence and demographic stochasticity in the evolution of cooperation
}

\author{
Thomas LoFaro \\ Department of Mathematics, Computer Science and Statistics, Gustavus Adolphus College, Saint Peter, MN, \\ USA
}

\begin{abstract}
We make two simplifications to a joint population model developed by M. Doebeli et al. of two populations whose growth rates depend on total population density and pay-offs governed by the Iterated Prisoner's Dilemma. One population uses the'Always Defect'strategy and the second uses the 'Tit for Tat' (TFT) strategy. In the deterministic model, there are two simple basins of attraction that lead to the extinction of one or the other population. In particular, a small TFT population cannot spread from rarity. We compute the boundary between these two regions. If, on the other hand, the growth rate of the TFT population is stochastic, then it is possible for the TFT population to become established if the growth rate at any given time is sufficiently large to allow the TFT population to cross the threshold computed in the deterministic model. We describe the factors that increase the likelihood of TFT establishment and explain why density dependence is an essential feature of the model. In particular, we show that if the relative advantage of defecting is small compared to the benefits of cooperating, then there is an increased likelihood that cooperation will evolve.
\end{abstract}

\section{ARTICLE HISTORY}

Received 21 July 2015

Accepted 5 October 2015

\section{KEYWORDS}

Cooperation; evolution; stochasticity

\section{Introduction}

In Population dynamics, demographic stochasticity and the evolution of cooperation (Doebeli, Blarer, \& Ackermann 1997) investigate the role of demographic stochasticity in the evolution of cooperation. In that work, they interpret the pay-offs of the Iterated Prisoner's Dilemma as Darwinian fitness and numerically show that conditions exist under which a small number of mutants using an inherently cooperative strategy can invade a population of pure defectors (i.e. individuals who never cooperate). They show that in the absence of demographic stochasticity the mutant cooperators can never invade, but that if stochasticity is incorporated in the model then invasion is possible.

Two strategies for the Iterated Prisoner's Dilemma are discussed by Doebeli et al. The first is the Always Defect (AD) strategy which is inherently non-cooperative. The second strategy, known as Tit-for-Tat (TFT), is cooperative in the sense that individuals adopting 


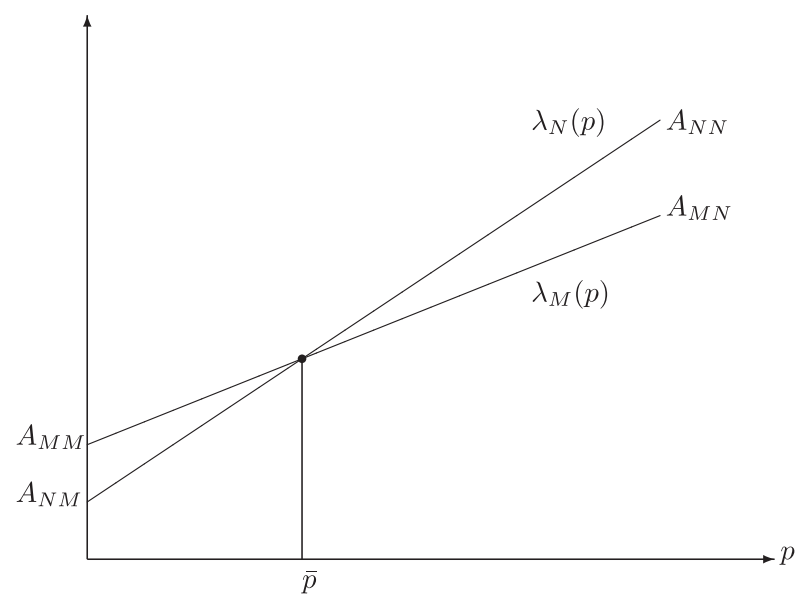

Figure 1. The lines $\lambda_{M}(p)$ and $\lambda_{N}(p)$.

Note: The $p$-coordinate of the intersection of these lines is denoted $\bar{p}$.

this strategy will cooperate with an opponent until that opponent chooses not to cooperate. In each successive meeting against that opponent, players adopting the TFT strategy will also defect. The authors investigate conditions under which invasion occurs and conjecture that cooperators 'can invade a population of defectors when their dynamics exhibit short episodes of high population densities with subsequent crashes and long low density periods with strong genetic drift'.

The purpose of this paper is to explain this conjecture and to show that the most salient feature of the model is not the population dynamics of the defectors, but rather the relationship between these dynamics, the location of a threshold dividing two basins of attraction and the variability of the cooperating population. In particular, we will show that this threshold is an increasing linear function of the defector population. Thus, invasion is more likely when the defector population is small. Moreover, the slope of this threshold can be expressed in terms of the Iterated Prisoner's Dilemma pay-off matrix. Thus, conditions for an increased likelihood of invasion can be expressed in game theoretic terms independent of population dynamics.

Section 2 discusses the basic deterministic model. This model is the foundation for all that follows and a thorough investigation of it provides a basis for understanding the more complex stochastic models. We will show that the line connecting the origin and a non-trivial fixed point is invariant and that this line forms the boundary between the two basins of attraction in the deterministic model.

In Section 3, we discuss a slightly simplified model of the one presented in Doebeli et al. (1997). We will examine several scenarios that illustrate the connections between the defector dynamics, the threshold and the variability of cooperating population. In particular, we compute how the probability of crossing the threshold changes with respect to both the slope of this threshold and the AD population size.

\section{The deterministic model}

We begin by summarizing the construction of the mathematical model as presented in Doebeli et al. (1997). However, we will relax the restriction made in that work that 


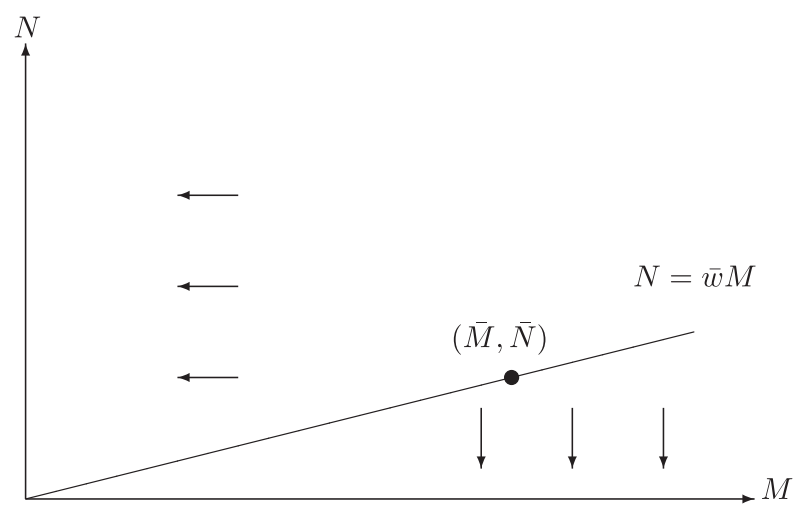

Figure 2. The $(M, N)$ plane and the line separating the two basins of attraction.

Notes: Orbits starting below the threshold converge to an attracting orbit on the $M$-axis while orbits starting above the threshold converge to an attracting orbit on the $\mathrm{N}$-axis.

populations take only non-negative integer values; allowing non-negative real values for these variables.

The Prisoner's Dilemma is a two-player game where players can adopt either a cooperative strategy $(\mathrm{C})$ or a non-cooperative strategy, denoted $\mathrm{D}$ for 'defect'. The pay-offs of this game are expressed in a pay-off matrix of the form

\begin{tabular}{c||c|c} 
& $\mathrm{C}$ & $\mathrm{D}$ \\
\hline \hline $\mathrm{C}$ & $R$ & $S$ \\
\hline $\mathrm{D}$ & $T$ & $P$ \\
\hline
\end{tabular}

where the pay-offs are received by the player in the first column playing against a player in the first row. For example, the pay-off to a player of type $C$ playing against an opponent of type $\mathrm{D}$ is $S$. In the Prisoner's Dilemma, the pay-offs are chosen so that $S<P<R<T$. It is well known that in this game the evolutionary stable strategy is to defect (strategy D) (Axelrod \& Hamilton, 1981).

The Iterated Prisoner's Dilemma game is built from this game by assuming that there is some non-zero probability that opponents will meet again. This repetition allows for more complex strategies that build upon the basic strategies of the Prisoner's Dilemma. The first strategy we will consider is AD. A player using this strategy always chooses option D. A more cooperative strategy is known as TFT. In this strategy, the player cooperates in the first round and then chooses the strategy that their opponent used previously in each subsequent meeting of the two. In a TFT vs AD matchup, TFT would cooperate in the first meeting and then defect in each subsequent meeting. However, in a TFT vs TFT matchup, each individual would cooperate in every round.

We consider a discrete-time model of two populations whose population growth depends upon both population density and the pay-offs of an Iterated Prisoner's Dilemma game. Let $M_{t}$ denote the $\mathrm{AD}$ population at generation $t$ and $N_{t}$ the TFT population at generation $t$. Because the Iterated Prisoner's Dilemma is played repeatedly, the pay-offs of this game depend on both the standard Prisoner's Dilemma pay-offs and the frequency at which opponents encounter each other. Axelrod and Hamilton (1981) and Doebeli et al. (1997) use a geometric series calculation to show that if the probability of repeated encounters is sufficiently large, then the pay-offs are given by 


\begin{tabular}{c||c|c} 
& $N$ & $M$ \\
\hline \hline$N(\mathrm{TFT})$ & $A_{N N}$ & $A_{N M}$ \\
\hline$M(\mathrm{AD})$ & $A_{M N}$ & $A_{M M}$ \\
\hline
\end{tabular}

where $A_{N M}<A_{M M}<A_{M N}<A_{N N}$. Note that the order of these inequalities is different from the standard Prisoner's Dilemma. In particular, the highest pay-off is in a TFT vs TFT match up where both players repeatedly cooperate. This is due to an assumption in Doebeli et al. (1997) that demands that the frequency of repeated encounters be relatively large. A consequence of this condition is that both $\mathrm{AD}$ and TFT are evolutionarily stable strategies (Axelrod \& Hamilton, 1981).

We interpret the pay-offs of the Iterated Prisoner's Dilemma as reproductive success. Because the population is heterogeneous, the average pay-off depends on the population frequency $p=N / Q$ where $Q=M+N$ is the total population. The average pay-offs are given by

$$
\begin{aligned}
& \lambda_{M}(p)=A_{M N} p+A_{M M}(1-p) \\
& \lambda_{N}(p)=A_{N N} p+A_{N M}(1-p) .
\end{aligned}
$$

The graphs of these functions are shown in Figure 1.

In addition to the game theoretic aspect of the model, we incorporate density dependence via a viability function $V(Q)$ that depends on the total population size. We assume that $V(Q)$ is a differentiable and monotone decreasing function with $V(0)=1$ and $\lim _{Q \rightarrow \infty} V(Q)=0$. In the simulations discussed below, we will take $V(Q)=e^{-c Q}$ where $c>0$.

Using these assumptions to construct our model, we get

$$
\begin{aligned}
M_{t+1} & =\lambda_{M}\left(p_{t}\right) V\left(Q_{t}\right) M_{t} \\
N_{t+1} & =\lambda_{N}\left(p_{t}\right) V\left(Q_{t}\right) N_{t} .
\end{aligned}
$$

This is essentially the same model discussed in Doebeli et al. (1997).

We begin our analysis by noting that if $N_{0}=0$, then $N_{t}=0$ for all $t$ and the system reduces to the one-dimensional map

$$
M_{t+1}=A_{M M} V\left(M_{t}\right) M_{t} .
$$

The dynamics and bifurcations of this family of maps are understood and similar to the dynamics of the logistic map $x_{n+1}=\lambda x_{n}\left(1-x_{n}\right)$. In this case, the dynamics depend on the parameter $A_{M M}$ and the density dependence function $V(M)$. As $A_{M M}$ increases, the dynamics range from a stable equilibrium, through the standard period-doubling cascade, to chaos and other stable periodic dynamics. Similar dynamics can occur when $M_{0}=0$ and in this case depend on the parameter $A_{N N}$.

Of special interest to us is that both of these orbits are transversally stable as well. This reflects the evolutionary stability of these two strategies. Thus, for example, if we have an initial condition of $\left(M_{0}, N_{0}\right)$ with $N_{0}$ sufficiently small, then $\lim _{t \rightarrow \infty} N_{t}=0$. To see this, let us consider a period $T$ orbit

$$
\left\{\left(M_{0}, 0\right),\left(M_{1}, 0\right), \ldots,\left(M_{T-1}, 0\right)\right\}
$$


and linearize about it. To compute the stability of this periodic orbit, we need to find the eigenvalues of the $T$-fold product of the Jacobian matrices of system (1) evaluated at each of these points. Each of these matrices is triangular so the product is also triangular and the eigenvalues are simply the diagonal elements of this product. In this case, the relevant eigenvalue is

$$
\mu=\lambda_{N}(0)^{T} \prod_{t=0}^{T-1} V\left(M_{t}\right)=A_{N M}^{T} \prod_{t=0}^{T-1} V\left(M_{t}\right) .
$$

Periodicity implies that

$$
M_{0}=A_{M M}^{T}\left[\prod_{t=0}^{T-1} V\left(M_{t}\right)\right] M_{0}
$$

which implies that

$$
\prod_{t=0}^{T-1} V\left(M_{t}\right)=\frac{1}{A_{M M}^{T}} .
$$

Substituting (3) into (2) implies that

$$
\mu=\left(\frac{A_{N M}}{A_{M M}}\right)^{T}<1
$$

since $A_{N M}<A_{M M}$ Thus, this orbit is asymptotically stable transverse to the $M$-axis. A similar calculation reveals that a periodic orbit on the $N$-axis is also asymptotically stable. We note that the periodicity assumption is not necessary but the proof requires the use of ergodic theory. For a similar proof, see LoFaro and Gomulkiewicz (1999).

Regardless of the axial dynamics, there also exists a non-zero equilibrium point $(\bar{M}, \bar{N})$ with $\bar{M}=\bar{Q}(1-\bar{p})$ and $\bar{N}=\bar{Q} \bar{p}$. To compute $\bar{p}$, we note that the Equations (1) imply that if there exists a fixed point, then $\lambda_{M}(\bar{p})=\lambda_{N}(\bar{p})$ and thus from (1) it follows that

$$
\bar{p}=\frac{A_{N M}-A_{M M}}{A_{M N}-A_{N N}+A_{N M}-A_{M M}} .
$$

The inequalities describing these parameters imply that $\bar{p}>0$. We note that $\bar{Q}$ can also be expressed in terms of these parameters (and the function $V$ ) but this will be irrelevant to what follows.

Linearization implies that the fixed point $(\bar{M}, \bar{N})$ is a saddle and it is this structure that separates orbits that tend to the $M$-axis from those that converge to the $N$-axis.

Proposition 2.1: $\quad$ Let $\bar{w}=\bar{p} /(1-\bar{p})$. The line $N=\bar{w} M$ is invariant. Moreover, if $N_{0}<\bar{w} M_{0}$, then

$$
\lim _{t \rightarrow \infty} N_{t}=0 .
$$

Similarly, if $N_{0}>\bar{w} M_{0}$, then

$$
\lim _{t \rightarrow \infty} M_{t}=0 .
$$



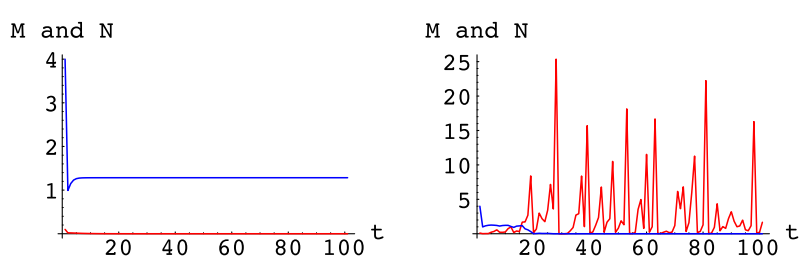

Figure 3. Deterministic and stochastic iteration.

Notes: In each figure, the horizontal axis is $t$ and the vertical axis is population with blue representing $M$ and red representing $N$. The same initial conditions were used to generate each figure. The figure on the left is the deterministic model. Note that the sequence $\left\{M_{t}\right\}$ converges to a non-zero value while the sequence $\left\{N_{t}\right\}$ converges to zero. The figure on the right is the stochastic model. In this example, the sequence $\left\{M_{t}\right\}$ becomes negligible.

Proof: Suppose that $N_{0}=\bar{w} M_{0}$. Then $Q_{0}=M_{0} /(1-\bar{p})$ and $p_{0}=\bar{p}$. This implies that

$$
\frac{N_{1}}{M_{1}}=\frac{\lambda_{N}(\bar{p}) N_{0}}{\lambda_{M}(\bar{p}) M_{0}}=\frac{N_{0}}{M_{0}}
$$

since $\lambda_{M}(\bar{p})=\lambda_{N}(\bar{p})$. Thus, the line $N=\bar{w} M$ is invariant.

We prove the second part of this proposition by showing that if $N_{0}<\bar{w} M_{0}$, then $p_{t} \rightarrow 0$ as $t \rightarrow \infty$. The equations in (1) imply that

$$
p_{t+1}=\frac{\lambda_{N}\left(p_{t}\right) V\left(Q_{t}\right) p_{t} Q_{t}}{Q_{t+1}}
$$

If $N_{t}<\frac{\bar{p}}{1-\bar{p}} M_{t}$, then $p_{t}<\bar{p}$ and thus $\lambda_{N}\left(p_{t}\right)<\lambda_{M}\left(p_{t}\right)$. Therefore

$$
\frac{p_{t+1}}{p_{t}}=\frac{\lambda_{N}\left(p_{t}\right)}{p_{t} \lambda_{M}\left(p_{t}\right)+\left(1-p_{t}\right) \lambda_{N}\left(p_{t}\right)}<\frac{\lambda_{N}\left(p_{t}\right)}{p_{t} \lambda_{N}\left(p_{t}\right)+\left(1-p_{t}\right) \lambda_{N}\left(p_{t}\right)}=1
$$

This implies that if $0<p_{0}<\bar{p}$, then the sequence $\left\{p_{t}\right\}_{t=0}^{\infty}$ is a strictly decreasing sequence and $\lim _{t \rightarrow \infty} p_{t}=0$. Thus, in the original equations if $N_{0}<\frac{\bar{p}}{1-\bar{p}} M_{0}$ then $\lim _{t \rightarrow \infty} N_{t}=0$.

An identical argument is used to prove the second limit.

Figure 2 summarizes the dynamics of the deterministic model. The $M$ - and $N$-axes are invariant and on each of these axes there exists an attracting orbit. These orbits may be periodic or chaotic. Moreover, these orbits are also transversally stable and so initial conditions off the axes that also converge to these orbits. The line $N=\bar{w} M$ is also invariant and orbits with initial conditions on this line converge to the fixed point $(\bar{M}, \bar{N})$. Proposition 2.1 tells us that this line also serves as the boundary between the basins of attraction of the attracting orbits on the $M$ - and $N$-axes. Orbits with initial conditions below this threshold line converge to the attracting orbit on the $M$-axis, while orbits starting above this line converge to the $\mathrm{N}$-axis. 

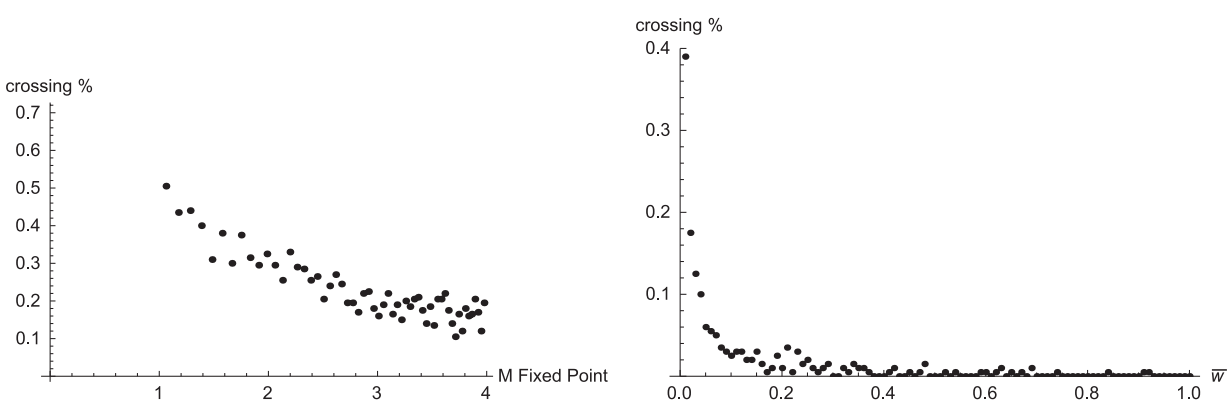

Figure 4. The probability of crossing the threshold as a function of the fixed point location $\left(M^{*}, 0\right)$ (left) and of the slope $\bar{w}$ (right).

Notes: Left: Parameters were chosen so that there is an attracting fixed point on the $M$-axis and the slope of the threshold is $\bar{w}=0.1$. An initial condition of $\left(M^{*}, 0.1\right)$ was used in all trials. Right: Parameters were chosen so that there is an attracting fixed point on the $M$-axis. An initial condition of $(3.219,0.02)$ was used for all trials. This initial condition has the same $M$-coordinate as the fixed point and is below the threshold for all values of $\bar{w}$.

\section{A stochastic model}

With a complete understanding of the deterministic model in hand, we can now begin investigating a similar model that incorporates stochasticity. We consider a model in which the only randomness is in the dynamics of the $N$ variable representing the TFT population. This is a simplification of the model discussed in Doebeli et al. (1997) where stochasticity affected the dynamics of both populations. We make this simplification so that we can better elucidate the factors that allow for the evolution of cooperation.

In the deterministic model, the growth rate at generation $t$ is the deterministic function $\lambda_{N}\left(p_{t}\right) V\left(Q_{t}\right)$. In this section, we assume that the growth rate at generation $t$ is drawn from a continuous probability distribution on the interval $(0, \infty)$ with mean $\lambda_{N}\left(p_{t}\right) V\left(Q_{t}\right)$.

The basic idea is that stochasticity allows for an orbit that would deterministically converge to the $M$-axis to cross the threshold (i.e. the line $N=\bar{w} M$ ). This might occur, for example, if the birth rate is unusually high in some generation. Once the threshold has been crossed, then it is possible that this orbit will then converge to the $N$-axis instead of the $M$-axis. Thus, stochasticity has provided an opportunity for cooperators to prevail in the face of competition. Figure 3 shows examples of the deterministic and stochastic model using the same parameter values and initial conditions. On the left is the deterministic model. Note that the sequence $\left\{N_{t}\right\}$ (in blue) converges to a non-zero value while the sequence $\left\{M_{t}\right\}$ (in red) converges to zero. Thus, TFT does not spread against AD. By contrast, the stochastic model with the same initial conditions has a markedly different behavior. In this example, the sequence $\left\{M_{t}\right\}$ does not converge to 0 and thus the TFT strategy persists. We note that because this model is stochastic, each run is different. In particular, we have observed runs using the same model where TFT does not invade in the 100 iterations shown here.

There are two main factors that facilitate the crossing of the threshold. The first is the variance of the probability distribution. A large variance will increase the likelihood that the threshold will be crossed.

The second and more fundamental factor is the location of the threshold relative to the attracting orbit on the $M$-axis. If the threshold is close to points on this orbit, then there is 
an increased probability that the stochasticity will push the $N$ orbit across the threshold. Since the threshold is the line $N=\bar{w} M$ which passes through the origin, one way that the threshold can be near to points on the attracting orbit on the $M$-axis is if points on the attracting orbit are close to the origin. This will allow relatively small deviations from the deterministic model to push orbits across the threshold and into the basin of attraction of the attracting orbit on the $\mathrm{N}$-axis. In terms of the model, this means that if the AD population experiences periods of low populations then there is an increased likelihood that the TFT population could invade. This is the mechanism suggested by Doebeli et al. (1997). Note that this mechanism does not require the $M$ dynamics to be chaotic or even periodic; it only requires that there be periods when this orbit (i.e. the $\mathrm{AD}$ population) is very small.

Another way to have a small distance between the attracting orbit on the $M$-axis and the threshold is for the slope of the threshold to be small. But this slope is

$$
\bar{w}=\frac{\bar{p}}{1-\bar{p}}=\frac{A_{M M}-A_{N M}}{A_{N N}-A_{M N}}
$$

which is solely a function of the IPD pay-offs. The numerator of this fraction is the difference in pay-offs in games against $\mathrm{AD}$, while the denominator is the difference in pay-offs in games against TFT. Thus, if the pay-off differential between AD and TFT in matches against $\mathrm{AD}$ (the numerator) is small relative to the pay-off differential between TFT and $\mathrm{AD}$ in matches against TFT (the denominator), then there is an increased likelihood that TFT 'mutants' will spread and ultimately cooperation will prevail. In other words, if it is only slightly advantageous to defect relative to the benefits of cooperating then cooperation may spread.

We now make these claims more specific. Recall that we have made the simplifying assumption that the $M$ dynamics are deterministic, while the $N$ dynamics are stochastic with a random growth rate having a mean of $\lambda_{M}\left(p_{t}\right) V\left(Q_{t}\right)$. Let $r_{\mu}$ denote a random variable chosen from a continuous probability distribution $R_{\mu}$ on $[0, \infty)$ with mean $\mu$. Then, the model equations become

$$
\begin{aligned}
M_{t+1} & =\lambda_{M}\left(p_{t}\right) V\left(Q_{t}\right) M_{t} \\
N_{t+1} & =r_{\mu_{t}} N_{t} .
\end{aligned}
$$

with $\mu_{t}=\lambda_{N}\left(p_{t}\right) V\left(Q_{t}\right)$.

Proposition 3.1: Let $P$ denote the probability of the next iterate of $\left(M_{t}, N_{t}\right)$ under (6) crossing the the threshold line $N=\bar{w} M$. Then $\partial P / \partial \bar{w}<0$ and $\partial P / \partial M_{t+1}<0$.

Proof: Using the notation above, we can express the probability of crossing the threshold in the next iteration for $\left(M_{t}, N_{t}\right)$ below the threshold. The next iteration crosses the threshold if and only if

$$
N_{t+1}>\bar{w} M_{t+1}
$$

Equivalently, if

$$
r_{\mu_{t}}>\frac{\bar{w} \lambda_{M}\left(p_{t}\right) V\left(Q_{t}\right) M_{t}}{N_{t}}
$$


then the threshold is crossed. Thus, the probability $P$ that the threshold will be crossed in the next iteration is given by

$$
P=\int_{\frac{\tilde{\omega} \lambda_{M}\left(p_{t}\right) V\left(Q_{t}\right) M_{t}}{N_{t}}}^{\infty} R_{\mu_{t}}(x) \mathrm{d} x .
$$

Since $\bar{w}$ is the slope of the threshold, we can determine how this affects the probability of crossing the threshold by taking the derivative of equation (7) with respect to $\bar{w}$. This yields

$$
\frac{\partial P}{\partial \bar{w}}=-R_{\mu_{t}}\left(\frac{\bar{w} M_{t+1}}{N_{t}}\right) \frac{M_{t}}{N_{t}} \lambda_{M}\left(p_{t}\right) V\left(Q_{t}\right)<0 .
$$

Therefore, the probability of crossing the threshold increases as $\bar{w}$ (or equivalently $\bar{p}$ ) decreases.

It is more difficult to determine how $P$ changes with $M_{t}$ since the dynamics of this variable may not be monotonic. However, we can easily compute the rate of change of $P$ with respect to $M_{t+1}$ (the image of $M_{t}$ ). The probability of crossing the threshold is

$$
\int_{\frac{\tilde{w} M_{t+1}}{N_{t}}}^{\infty} R_{\mu_{t}}(x) \mathrm{d} x
$$

Thus, the rate of change of $P$ with respect to $M_{t+1}$ is

$$
\frac{\partial P}{\partial M_{t+1}}=-R_{\mu_{t}}\left(\frac{\bar{w} M_{t+1}}{N_{t}}\right) \frac{\bar{w}}{N_{t}}<0 .
$$

Thus, as the value of $M_{t+1}$ decreases the probability of crossing the threshold increases. In other words, if the AD population crashes, then the probability of crossing the threshold increases and hence there is an increased likelihood that the TFT population will become established.

Explicit computations and simulations can be made when the probability distribution $R$ is assumed to be the exponential distribution. This is a natural distribution to use in this work given that the stochasticity is assumed to play a role in determining the growth rate of the TFT population. A Poisson distribution was used in this context in Doebeli et al. (1997) since they restricted the values of $M$ and $N$ to the positive integers. Recall that the exponential distribution is a one-parameter probability distribution defined on the interval $[0, \infty)$ and for a fixed parameter $\lambda$

$$
\operatorname{Pr}(x>A)=\int_{A}^{\infty} \lambda e^{-\lambda x} \mathrm{~d} x=e^{-\lambda A} .
$$

The mean of this distribution is $\lambda^{-1}$ and its variance is $\lambda^{-2}$.

We begin with a pair of numerical simulations (see Figure 4) using Mathematica (Wolfram Research, Inc., 2015) to illustrate how the location of the asymptotic orbit on the $M$-axis and the threshold slope affects the probability of crossing the threshold. To highlight the effect of the threshold slope and to eliminate the other factors we have discussed, we have 
chosen model parameters so that there is an attracting equilibrium $\left(M^{*}, 0\right)$ for all trials. This fixed point is given by $M^{*}=\ln \left(A_{M M}\right) / c$ and is attracting for $1<A_{M M}<e^{2}$.

In the first simulation (left panel of Figure 4), we fixed the slope $\bar{w}=0.1$ and increased $A_{M M}$ from 1 to $e^{2}$ in steps of 0.1 . An initial condition of $\left(M^{*}, 0.1\right)$ was used in all trials. We let $A_{M N}=14, A_{N N}=15, c=0.5$ and $A_{N M}=A_{M M}-0.1$. In all simulations, we conducted 200 trials allowing a maximum of 100 iterations per trial. We plot the number of trials in which the threshold was crossed at least once divided by the number of trials. Figure 4 suggests that the probability of crossing the threshold decreases as $M^{*}$ increases as discussed above.

In the second simulation (right panel of Figure 4), we fix the location of the fixed point throughout the trials and we change the slope $\bar{w}$ by changing the IPD pay-off $A_{N M}$ while leaving $A_{M M}, A_{N N}$ and $A_{M N}$ fixed. For Figure 4, we let $A_{M M}=5, A_{M N}=14, A_{N N}=15, c=0.5$ and $A_{N M}=A_{M M}-\delta$ with $\delta$ ranging from 0.01 to 1.0 in steps of 0.01 . Note that these choices of parameters imply that $M^{*} \approx 3.219$ and $\bar{w}=\delta$. An initial condition of $(3.219,0.02)$ was used for all trials. In all simulations, we conducted 200 trials allowing a maximum of 100 iterations per trial. We plot the number of trials in which the threshold was crossed at least once divided by the number of trials. Again we see that the probability of crossing the threshold decreases as $\bar{w}$ increases as is predicted by Proposition 3.1.

While we can explicitly compute the derivatives described in Proposition 3.1 using the exponential distribution, the simplicity of Equation (9) allows for the direct computation of both $\partial P / \partial M_{t}$ and $\partial P / \partial N_{t}$ for which we do not have results in general. For ease of notation, we will drop the subscript $t$ in what follows. First note that when using the exponential distribution the probability of crossing the threshold is

$$
P=\exp \left(-\frac{M\left(A_{M M} M+A_{M N} N\right)}{N\left(A_{N M} M+A_{N N} N\right)} \bar{w}\right) .
$$

Using this, a straightforward computation yields

$$
\frac{\partial P}{\partial M}=-\bar{w} P \frac{A_{M N} A_{N N} N^{2}+2 A_{M M} A_{N N} M N+A_{M M} A_{N M} M^{2}}{N\left(A_{N M} M+A_{N N} N\right)^{2}}<0
$$

and

$$
\frac{\partial P}{\partial N}=\bar{w} P \frac{A_{M N} A_{N N} M N^{2}+2 A_{M M} A_{N N} M^{2} N+A_{M M} A_{N M} M^{3}}{N^{2}\left(A_{N M} M+A_{N N} N\right)^{2}}>0 .
$$

Equation (11) is not surprising as it says that as we increase $N$ towards the threshold the probability of crossing the threshold increases. Equation (10) is less obvious since the dynamics when $N=0$ may be non-monotone. It says that as $M$ increases the probability of crossing the threshold in the next iteration decreases.

\section{Conclusion}

Two simplifications of the original model were made to facilitate mathematical analysis. The first modification, allowing $M$ and $N$ to assume real values instead of integer values, allowed the use of tools and techniques of differentiable dynamical systems. In particular, when 
studying the discrete model in Section 2, it allowed us to compute the stability of orbits on $M$ - and $\mathrm{N}$-axes, compute the invariant threshold line and ultimately to determine basins of attraction. The second simplification, allowing only the $N$ dynamics to vary stochastically, was done to simplify the calculation of the probability of crossing the threshold and the related rates of change. These simplifications provide a more complete mathematical and theoretical explanations of the phenomena originally described in Doebeli et al. (1997) and thus provide a foundation for pursuing a more complete mathematical description of the results described there.

A next step in understanding the simplified model is to describe the interplay between the asymptotic dynamics on the $M$-axis (the $\mathrm{AD}$ dynamics) and the stochastic dynamics of the $N$ variable (the TFT dynamics) in a neighborhood of the $M$-axis especially when the $M$-dynamics are periodic or chaotic. Ergodic theory tells us that there is an invariant probability measure associated with the invariant dynamics on the $M$-axis. Thus for the deterministic model, we can essentially 'compute' the asymptotic frequency (i.e. probability) that an orbit spends in some measurable set $S$ including a measurable invariant set on the $M$-axis. On the other hand, for each point in $S$, we can compute the probability of crossing the threshold using Equation (7). That suggests a possible mechanism for understanding how the underlying dynamics of the AD population affects the probability of the TFT population becoming established.

We have shown that in this model a small population of TFT mutants can become established when there is a stochastic component to their population growth rate. Moreover, we have explained why this invasion is more likely when, in the absence of a TFT population, the $\mathrm{AD}$ population is small and at equilibrium or experiences periods of low population densities. The explanation of this phenomenon in terms of the threshold line justifies the results presented in Doebeli et al. (1997).

By explicitly computing this threshold, we have also shown that invasion by TFT mutants is more likely when the relative advantage of defecting is small compared to the benefits of cooperating. We note that these two factors can act in concert to increase the likelihood of invasion.

It is worth noting that the density-dependent viability term included in this model is necessary. Without density dependence, both the TFT and AD strategies are evolutionary stable. In the deterministic model without density dependence, orbits near one of the axes still converge to that axis. However, the absence of density dependence causes the orbits on each axis to increase without bound. Thus, when stochasticity is incorporated in the model, there is little chance for the TFT mutants to become established. If the TFT population fails to cross the threshold in one generation, then the AD population will increase in the next generation and the probability of crossing the threshold diminishes. In the limit the probability of escape tends to zero.

Thus it seems that the evolution of cooperation depends critically on density dependence and randomness. Without either of these factors, it is impossible for cooperation to become established in the framework of this model.

\section{Disclosure statement}

No potential conflict of interest was reported by the author. 


\section{References}

Axelrod, R., \& Hamilton, W. D. (1981). The evolution of cooperation. Science, 211, 1390-1396.

Doebeli, M., Blarer, A., \& Ackermann, M. (1997). Population dynamics, demographic stochasticity, and the evolution of cooperation. Proceeding of the National Acadamy of Sciences, USA, 94, 5167-5171.

LoFaro, T., \& Gomulkiewicz, R. (1999). Adaptation versus migration in demographically unstable populations. Journal of Mathematical Biology, 38, 571-584.

Wolfram Research, Inc. (2015). Mathematica (version 10.2 edition). Champaign, IL: Author. 